$61-64$

\title{
水生高等植物马来眼子菜和金鱼藻的 初级生产率(黑白瓶法)
}

\author{
章宗涉 张扬东

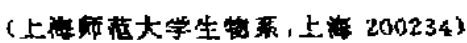

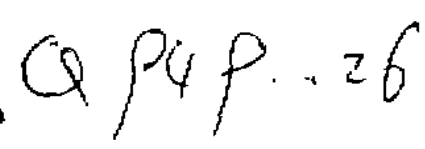

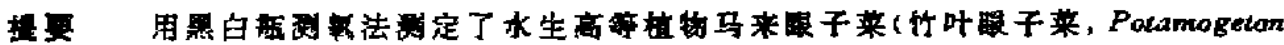

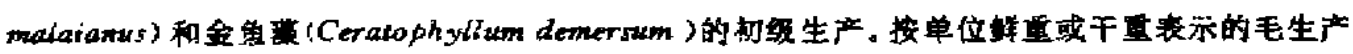

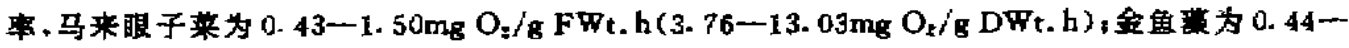

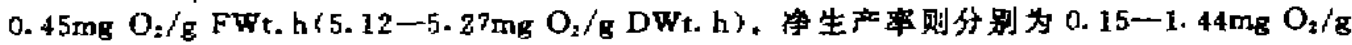

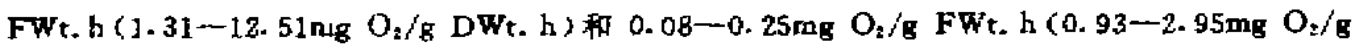

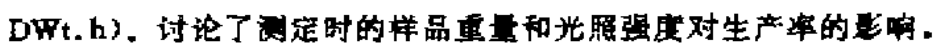

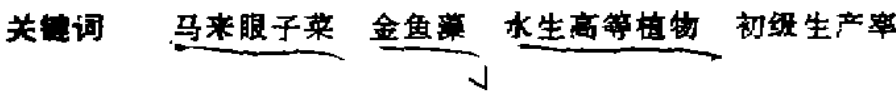

水体中初级生产的测定和研究，大量报道是关于浮游植物的,而对水生高等植物则研究 很少。我们对两种常见的水生高等植物一一古来眼子莱(竹叶眼子莱, Potamogeton

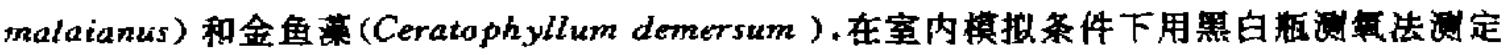
它们的光合产算和呼吸耗弹，计算了按单位鲜重或干重表示的生产来。

\section{材料和方法}

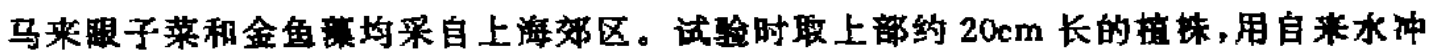

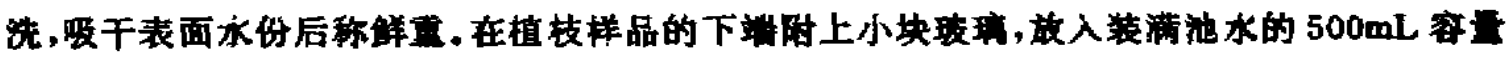

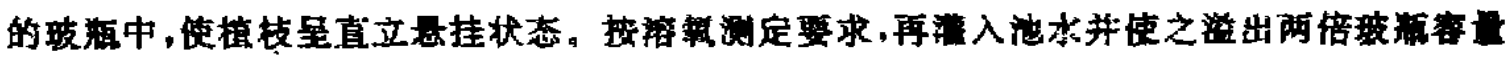

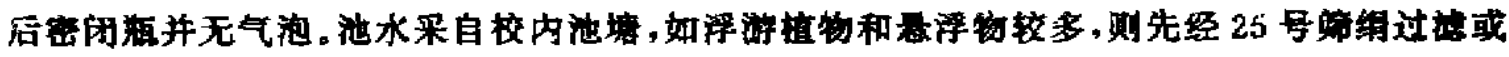
竟沸。

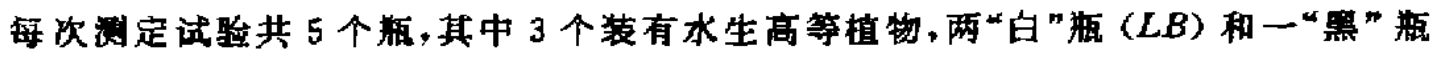

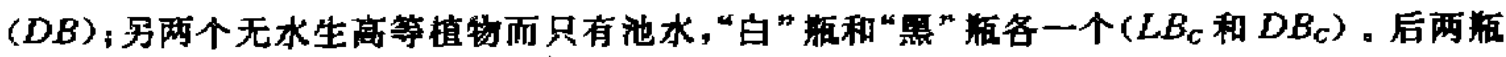
相当于“空白”，在计算水生高等植物生产率时用于扣除因池水中游植物和其他生物和非

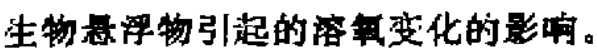

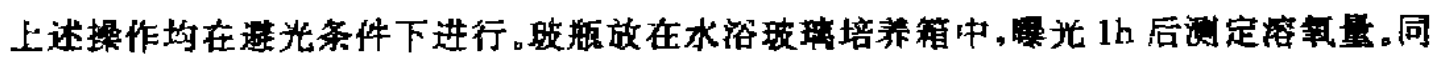


时测定了水生高等植物的鲜重与干重之比( $\mathrm{FW} t / \mathrm{DW} t) 。$

生产率 $\left(\mathrm{mg} \mathrm{O}_{2} / \mathrm{g} \mathrm{FWt.} \mathrm{h}\right.$ 或 DWt. h) 的计算方法为:

毛生产率 $\quad P_{\mathrm{a}}=(L B-D B)-\left(L B_{\mathrm{C}}-D B_{\mathrm{C}}\right)$.

净生产率 $\quad P_{\mathrm{n}}=L B-L B_{\mathrm{C}}$ 。

试验于 1989 年 4 月进行。

\section{结果 和 讨 论}

经测定,马来眼子菜的干重与鲜重之比平均为 $11.51 \%$, 金鱼落为 $8.59 \%$ 。 两种植物的生产率(按单位鲜重和千重计)见表 1 和表 2 。

衰 1 马来眼予莱的生产审

Tab. 1 Production rates of P. malalanus

\begin{tabular}{|c|c|c|c|c|c|}
\hline & 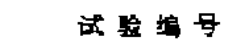 & 1 & 2 & 3 & 4 \\
\hline \multirow{2}{*}{$P_{x}$} & (mg Ozig DWt.h) & 7.22 & 9. 31 & 3. 76 & 13.03 \\
\hline & $\left(\mathrm{mg} \mathrm{O}_{2} / \mathrm{g} F \mathrm{Ft} \mathrm{ch}\right)$ & 0.83 & 1.07 & 0.43 & 1.50 \\
\hline \multirow{2}{*}{$P$} & $\left(\mathrm{mg} \mathrm{O}_{\mathrm{z} / \mathrm{g}} \mathrm{DW} \mathrm{t}, \mathrm{h}\right)$ & 6.74 & 7.75 & 1.31 & 12.51 \\
\hline & $\left\{\mathrm{mg} \mathrm{O} \mathrm{O}_{z} / \mathrm{g} F W_{\mathrm{L}} . \mathrm{h}\right)$ & 0.78 & 0.89 & 0.15 & 1.44 \\
\hline \multicolumn{2}{|c|}{$P_{n} / P_{n}$} & 0.94 & 0.83 & 0.34 & 0.96 \\
\hline \multicolumn{2}{|c|}{ 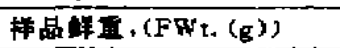 } & 0.86 & 0.43 & 1.43 & 0.80 \\
\hline \multicolumn{2}{|c|}{ 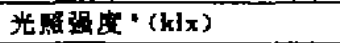 } & 7.5 & 7.5 & 7.5 & 21.3 \\
\hline \multicolumn{2}{|c|}{ 水酉( $\mathrm{t}$ C) } & $20-21$ & $22-23$ & 23 & $24-24.5$ \\
\hline
\end{tabular}

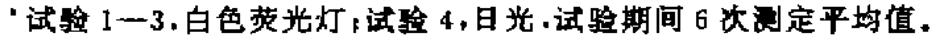

四次测定马来眼子菜的毛生产率为 $0.43-1.50 \mathrm{mg} \mathrm{O} / \mathrm{g} \mathrm{FWt}$. h 或 $3.76-13.03 \mathrm{mg}$ $\mathrm{O}_{2} / \mathrm{g}$ DWt.h。

椬物的光合作用与光强有密切关系，我们虽没有专门进行不同光强对光合作用的影响 的试验、但从试验 1 和试验 4 可看出.在样品鲜重、水温等试验条件基本相似时.由于光强不

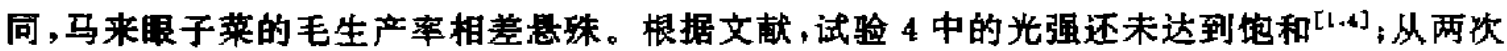

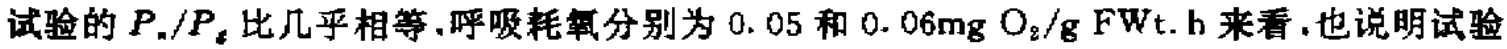
4 中的光强低于饱和光强。因此.光合作用随光强增高而增高.而呼吸耗氧则因样品重量相 近而几乎一样。

试验 1,2 和 3 中、光强和水温基本相同,但由于样品重量不同, 毛生产率也不同. 呈反比 关系(图 1)。Ikusima ${ }^{[3]}$ 也曾观察到类似现象。他认为其原因可能是由于样品重量增加。有遮 光作用；还可能有 $\mathrm{CO}_{2}$ 不足等方面的影响，因面使光合作用降低。

关于马来眼子菜的生产率,报道极少。Ikusima ${ }^{[\mathrm{w}}$ 在日本㖩访湖用黑白瓶法进行测定。通 过与光照强度关系的计算公式求得光饱和时的光合率为 $18.1 \mathrm{mg} \mathrm{O} / \mathrm{g} \mathrm{DWt.h}$ (水温 $25 \pm$ $2 \mathrm{C}$ )。我们的试验 4 中，光强尚未达到饱和 (见上).光合率为 $.13 .03 \mathrm{mg} \mathrm{O} / \mathrm{g} \mathrm{DWt}$. l . 是与之 可比的。 


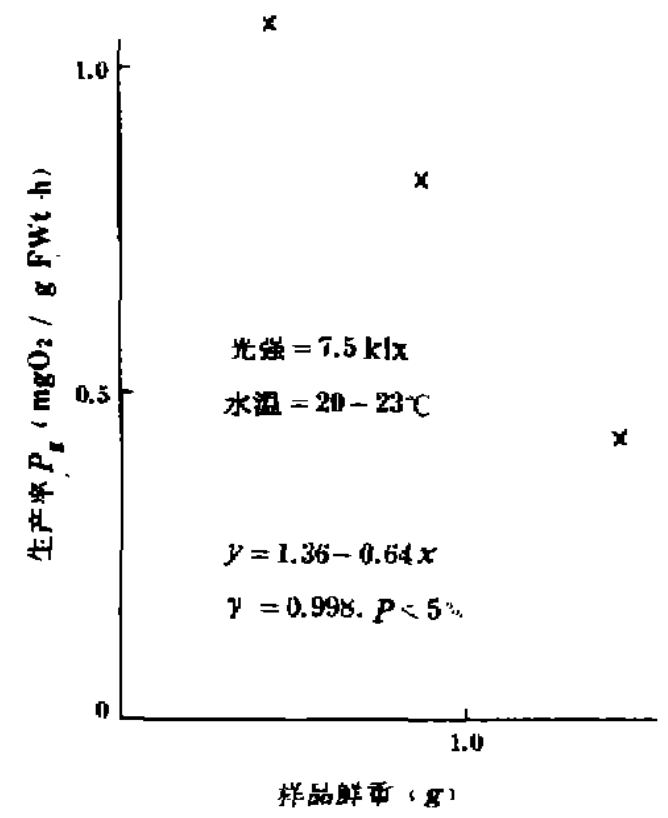

图 1 马来眼子莱的毛生产率与河定时样品徉重的关系

Fig. 1 The relationship between production rates $\left(P_{\&}\right)$ of $P$. malaianus and fresh weight of samples during measurement

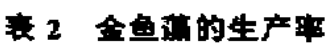

Tab. 2 Production rales of $C$. demersum

\begin{tabular}{|c|c|c|c|}
\hline & 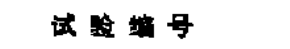 & 5 & 6 \\
\hline \multirow{2}{*}{$P}$. & ( $\left.\mathrm{mg} \mathrm{O}_{2} / \mathrm{g} \mathrm{DWt} \mathrm{h}\right)$ & 5.27 & 5.12 \\
\hline & $\left(m_{g} O_{s} / g F W h\right)$ & 0.45 & 0.44 \\
\hline \multirow{3}{*}{$P_{n}$} & $\left(\mathrm{mg} \mathrm{O}_{3} / \mathrm{g} \mathrm{DWt} h\right)$ & 2.95 & 0.93 \\
\hline & $\left(\mathrm{mg} \mathrm{O}_{\mathrm{g}} / \mathrm{g} \mathrm{FWt} \mathrm{h}\right)$ & 0.25 & 0.08 \\
\hline & 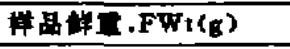 & 2.65 & 2.40 \\
\hline & 光照 & 3.5 & $5-3$ \\
\hline & 水淂 $\{r C\}$ & 23 & 21 \\
\hline
\end{tabular}

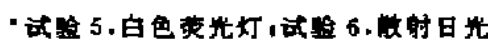

与風于同一風的蕰草 (P. crispus) 相比, 陈 洪达 ${ }^{[1]}$ 采用黑白瓶测氧法, 当样品重为 $0.5 \mathrm{~g}$ 鲜重. 光强为 $8 \mathrm{klx}$ ，水温为 $20-28^{\circ} \mathrm{C}$ 时, 平均 毛生产事为 $1.84 \mathrm{mg} \mathrm{O} / \mathrm{g}$ FWt. $\mathrm{h}$, 我们试验 2 的条件与之相近,马来眼子菜的毛生产事 为 $1.07 \mathrm{mg} \mathrm{O} \mathrm{O}_{2} / \mathrm{g} \mathrm{FWt}$. h。君来, 马来眼子菜 的光合事低于蕰草。

金鱼藻的生产率只测定了两次(表 2)， 毛生产事两次较接近、而净生产事差别较大。 文献报道金鱼藻的光合率差异也较大, 低的 不到 $1 \mathrm{mg} \mathrm{O} \mathrm{O}_{2} / \mathrm{g}$ DWt. h，最高的达到 $20 \mathrm{mg}$ $\mathrm{O}_{2} / \mathrm{g} \mathrm{DWt.h} \mathrm{h}^{[2.3 .5]}$ 。由于测定的条件和方法不

同，我们的湖定次数不多，难以作比较和讨论。

比较我们对马来眼子菜和金鱼落光合能力的测定结果,试验 3 和试验 5 中光照和温度 件相同,金鱼落的毛生产事在样品重量较大(光合率应偏低)的情况下.其毛生产率高于马 眼子菜。

植物的光合率还与其生长季节、生理状况及其他因素有关.这方面的问题将另文探讨。 


\title{
坴考文㩘
}

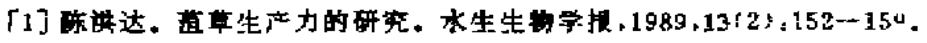

[2] Best E. P.H. ,J. T. Meulemana, Frimary productivity in the submerged aquatic macrophyte Ceratophydum demersum. Verh. Snternat. Verein. Limaut., 1976, 20:68-33.

[3] Ikusima I. Ecological studies on the productivity of aquatic pramt communities I. Mensurement of photouynthetic ativity. Bot, Mag. Tokyo, $1955,78: 202-211$.

[4] Ikusima I. , Ecological studies on the productivity of aquatic plant comamunities W. Light condituon and community photoeynthetic production. Bat, Mag. Tokyo, 1970,83,330-341.

[5] Lecren E. D. , R. H. Lowe-Mc Connell (ed.) . The functioning of freshwater ecosystems. Carnbridge University Press, 1980.574.

[6] Welzel R.G. ,Primery productivity of aquatic macrophytes. Verh. Internat. Vereiz. Lummoh., 1964,15,426-436.

\section{PRIMARY PRODUCTION RATES OF AQUATIC MACROPHYTES POT AMOGETON MALAIANUS AND CERATOPHYLLUM DEMERSUM, DETERMINED BY "LIGHT-DARK BOTTLE" METHOD}

\author{
Zhang Zongshe Zhang Yangdong \\ (Deperament of Bialogy, Shenghai A'drmal Untersity, Shanghai 200254)
}

\begin{abstract}
Abstrict
The primary production rates of aquatic macrophytes potamogeton maluiarus and ceratophylum demersw" were messured by "light-dark bottle" oxygen method. The grass primary production rates on the basis of unit fresh or dry weight for P. malaiams are C. 43-1. 50mg O./g FWh. h(3. 76-13.03mg Oz/S DWt. $h$ ), and for C. demersum $0.44-0.45 \mathrm{mg}_{2} / \mathrm{g} F \mathrm{Wt} . \mathrm{h}\left(5.12-5.27 \mathrm{mg} \mathrm{O}_{2} / \mathrm{g} \mathrm{DWt}\right.$. $\left.\mathrm{h}\right)$. The ret produc-

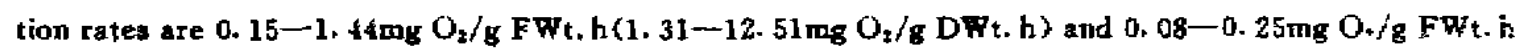
(0. 93-2. $95 \mathrm{mg} \mathrm{O}_{2} / \mathrm{g}$ DW $\mathrm{t} . \mathrm{h}$ ) respectivly. The effects of sample weight and light intensity on the measurement of production rate were discussed.
\end{abstract}

Key mords Palamogeton malaianus, caraphylizm demersum, aquatic macrophytes, primary production 